\title{
Performance of Adaptive MMSE Receivers Using Aided Tentative Coefficients in Flat Fading Channels
}

\author{
Khairy A. Elbarbary *
}

\begin{abstract}
The conventional matched filter (MF) receiver is considered the optimum filter to recover the CDMA signals. One of the problems of the MF is that its performance is significantly degraded due to the channel impairments and the increase of the multiple access interference (MAI). In this paper, an adaptive minimum mean square error-maximum likelihood (MMSE-ML) receiver is introduced to overcome this problem. This receiver uses two sets of adaptive coefficients to increase the ability of tracking the time variations of the channel. The performance of the receiver is measured in terms of bit error rate (BER) and compared with other receivers over flat fading channel. It is found that the performance of the adaptive MMSE-ML receiver is much better than the performance of the other receivers in the flat fading channel.
\end{abstract}

Keywords: code division multiple access (CDMA), minimum mean square error-maximum likelihood (MMSE-ML), matched filter (MF)

\section{Introduction:}

The code division multiple access (CDMA) is the technique, which enables more than one user to access the same channel on the same frequency at the same time. This process is performed by assigning a certain code with specific properties to each user. At the receiver, the data of each user is separated and recovered using this code. The optimum receiver that can perform this process is the matched filter (MF) receiver. A bank of matched filters is needed, where each MF is independent from others, each followed by a corresponding threshold detector to decode and classify the received symbols. MF has two major shortcomings: 1) very low near-far resistance and 2) very low information capacity, which is limited by multiple access interference (MAI) and multipath rather than thermal AWGN. The major advantage of the MF receiver is its low computational complexity and simple implementation.

Recently, the number of interfering user is hugely increased, that make the task of the MF more difficult especially in presence of the other channel impairments such as the colored additive Gaussian noise, fading, multipath,... etc.

\footnotetext{
* Egyptian Armed Forces
} 
Many researches are concentrated on the alternative solutions. A comparison of different types of receiver structures based on a bank of matched filters (MF's) has been derived, analyzed, and compared in [1]. They include the MF receiver, CDMA Decorrelator receiver (MF-DEC), MMSE receiver (MF-MMSE), and CDMA decision feedback receiver (MFDFE). The comparison including signature waveform and timing of the desired and interfering users, received amplitudes, and the training sequence of the desired user.

Although in a mobile radio channel, the MF bank statistics based receivers are important from the conceptual point of view, the following problems make the implementation of these receivers a difficult task.

1) The contributions from the variable number of users (interferers) in the single cell (which might be known), in addition to the other unknown users form the other cells.

2) The uncertain associated transmission delays of the users, and how it can be measured, as well as the associated uncertain signal power in addition to the interference power. While the intracellular interferers may be kept under precise power control, interferers from other cells cannot be controlled precisely.

3) The presence of colored additive Gaussian noise, or narrow-band interference, requires additional measures separate from the MF bank, while the adaptive MMSE filter bank removes this problem inherently [2].

In order to overcome the problem of CDMA system parameter estimation, adaptive receivers have been proposed, which require training sequence, at least for the initial period, so that the receiver converges to its steady state, and thereafter it can be made to run in a decision directed mode. An adaptive linear MMSE receiver is particularly attractive from the implementation point of view [2]-[5]. Its computational complexity is similar to the MF receiver, yet its performance (BER, near-far resistance, information capacity) is identical to the MF-MMSE receiver. Adaptive decision feedback equalizer (DFE) receivers (MMSEDFE) are considered in [4], [6], and [7]. They perform better than adaptive linear MMSE receivers do specially in case of unequal data rates [8], [9].

In this paper, we introduce a model of an adaptive MMSE-ML receiver using adaptive aided tentative coefficients in addition to the main adaptive coefficients. The performance of this receiver is compared with the traditional adaptive MMSE receiver that uses only the main adaptive coefficients. Moreover, the performances of both the adaptive receivers are compared with the SUMF, which is taken as a performance reference, in addition to the MF performance in the same conditions. The simulation is performed in presence of different number of MAI in flat fading channel. The adaptation process is performed using the least mean square (LMS) algorithm. It is shown that, the performance of the adaptive MMSE-ML receiver is better than the performance of the traditional adaptive MMSE receiver. Moreover, the performance of the adaptive MMSE-ML receiver converges to the performance of the MF with avoiding making the parameters estimation that represent the problem facing the MF in case of existing multiple access interference and fading. The adaptive implementation is simple and has the same computational complexity of the MF.

This paper is organized as follows. In Section 2, the structure of the adaptive MMSE-ML receiver is presented, and the adaptive solution of the MMSE-ML bank is discussed. The performance of the adaptive MMSE-ML receiver is presented in section 3. In Section 4, numerical results are presented showing the performance of the adaptive MMSE-ML receiver and compared with the adaptive MMSE, and the MF receiver. Last, Section 5 summarizes our study. 


\section{Adaptive MMSE-ML Receiver}

In this section, we introduce the adaptive MMSE-ML receiver. First, the problem of the MF in CDMA signal detection is presented. The asynchronous CDMA signal in additive white Gaussian noise can be expressed as

$$
y(t)=\sum_{k=1}^{K+K_{1}} \sum_{i=0}^{M-1} b_{k}(i) s_{k}\left(t-i T-\tau_{k}\right)+n(t)
$$

where $K$ is the number of users in the cell of interest, $K_{1}$ is number of non negligible interferers from other cells, $s_{k}(t)$ is the received signature waveform of the $k^{\text {th }}$ user. $b_{k}(i)$ is the $i^{\text {th }}$ symbol of the kth user, $\tau_{k}$ is the transmission delay of the $k^{\text {th }}$ user, $T$ is the inverse data rate, and $n(t)=\sigma w(t)$,where $w(t)$ is normalized white Gaussian noise and $\sigma$ is the noise variance. In the absence of intercellular interference $K_{1}=0$ the optimum MF receiver passes the signal $y(t)$ through a bank of matched filters and samples the outputs as

$r_{k}(i)=\int_{i T+\tau_{k}}^{(i+1) T+\tau_{k}} y(t) s_{k}(t-i T-\tau) d t, \quad 0 \leq i \leq M-1$

It is clear from (2) that $r_{k}(i)$ require perfect synchronization of $s_{k}(t)$ and $\tau_{k}$ of each user. In other words, equation (2) shows the difficulty of recovering the data from the asynchronous CDMA system using the MF. This is because it need to perfect synchronization and knowledge about the assigned code and the transmission delay of each user, especially with the difference between the received user signature $s_{k}(t)$, and transmitted used signature $f_{k}(t)$, where the relation between $s_{k}(t) \& f_{k}(t)$ is the convolution relation which is $s_{k}(t)=f_{k}(t) * \gamma_{k}(t)$, and $\gamma_{k}(t)$ is the channel impulse response. In case of flat fading channel $\gamma_{k}(t)$ is equal to constant random variable. Therefore, the degradation in performance of MF receiver is caused due to the Errors in estimation of $\gamma_{k}(t)$.

The adaptive MMSE-ML receiver structure overcomes these difficulties; it consists of a bank of $K$ adaptive fractionally spaced MMSE finite impulse response (FIR) filters along with the ML detector part of the receiver for data detection. The number of intercellular interference $K_{1}$ is unknown and only $K$ input MMSE filters interferers are used in the presented receiver. The output of the MMSE filter (actual output) at the $n^{\text {th }}$ symbol interval for the $k^{\text {th }}$ user is

$a_{k}(n)=\sum_{m=-P}^{P} c_{k}(m) y\left(n T-m T_{f}\right)$

where $c_{k}(m)$ are the adaptive filter coefficients, $T_{f}=T_{c} / p$ with, $p>1, T_{c}$ being the chip interval. The total number of adaptive filter coefficients is $(2 P+1)>p N$ where $N$ is the spreading gain. The symbol estimate is obtained as

$$
\hat{b}_{k}(n)=a_{k}(n)-\mathbf{d}_{k}^{H} \mathbf{b}_{k}
$$


where $\mathbf{d}_{k}$ is a vector of tentative decision aided coefficient sequences, defined as

$$
\mathbf{d}_{k}=\left[\mathbf{d}_{k 1}^{T}, \mathbf{d}_{k 2}^{T}, \mathbf{d}_{k 3}^{T}, \ldots \ldots . . . \mathbf{d}_{k K}^{T}\right]^{T}
$$

with $\mathbf{d}_{\mathbf{K m}}=\left[d_{k m}(0), \ldots \ldots \ldots d_{k m}(M-1)\right]^{T}$. The elements of $\mathbf{d}_{\mathbf{K m}}$ represent the aided tentative weighting coefficients, which multiply the respective known symbols coming from the $m^{\text {th }}$ user. The vector $\mathbf{b}_{k}$ contains known symbols to the receiver. It is defined as

$$
\mathbf{b}_{k}=\left[\mathbf{b}_{k 1}^{T}, \mathbf{b}_{k 2}^{T}, \mathbf{b}_{k 3}^{T}, \ldots \ldots . . . \mathbf{b}_{k K}^{T}\right]^{T}
$$

with $\mathbf{b}_{\mathrm{Km}}=\left[b_{k m}(0), \ldots \ldots \ldots b_{k m}(M-1)\right]^{T}$. The coefficients $\mathbf{d}_{k}$ and $\left\{c_{k}\right\}$ are obtained adaptively during a training period. After the training period, the coefficients $\mathbf{d}_{k}$ and $\left\{c_{k}\right\}$ can be kept fixed during data detection. Alternatively, in a decision directed mode, these coefficients can be updated by tentative decisions.

The filter coefficients are obtained by minimizing the MSEE[ $\left[\left.e_{k}(n)\right|^{2}\right]$, where $e_{k}(n)=b_{k}(n)-\hat{b}_{k}(n)$ is the error between the desired and actual outputs. There is a wide range of adaptive algorithms [10] to obtain the optimal coefficients $\mathbf{d}_{k}(n)$ and $\mathbf{c}_{k}(n)$. For them, we select the LMS algorithm. The following steps correspond to the LMS algorithm:

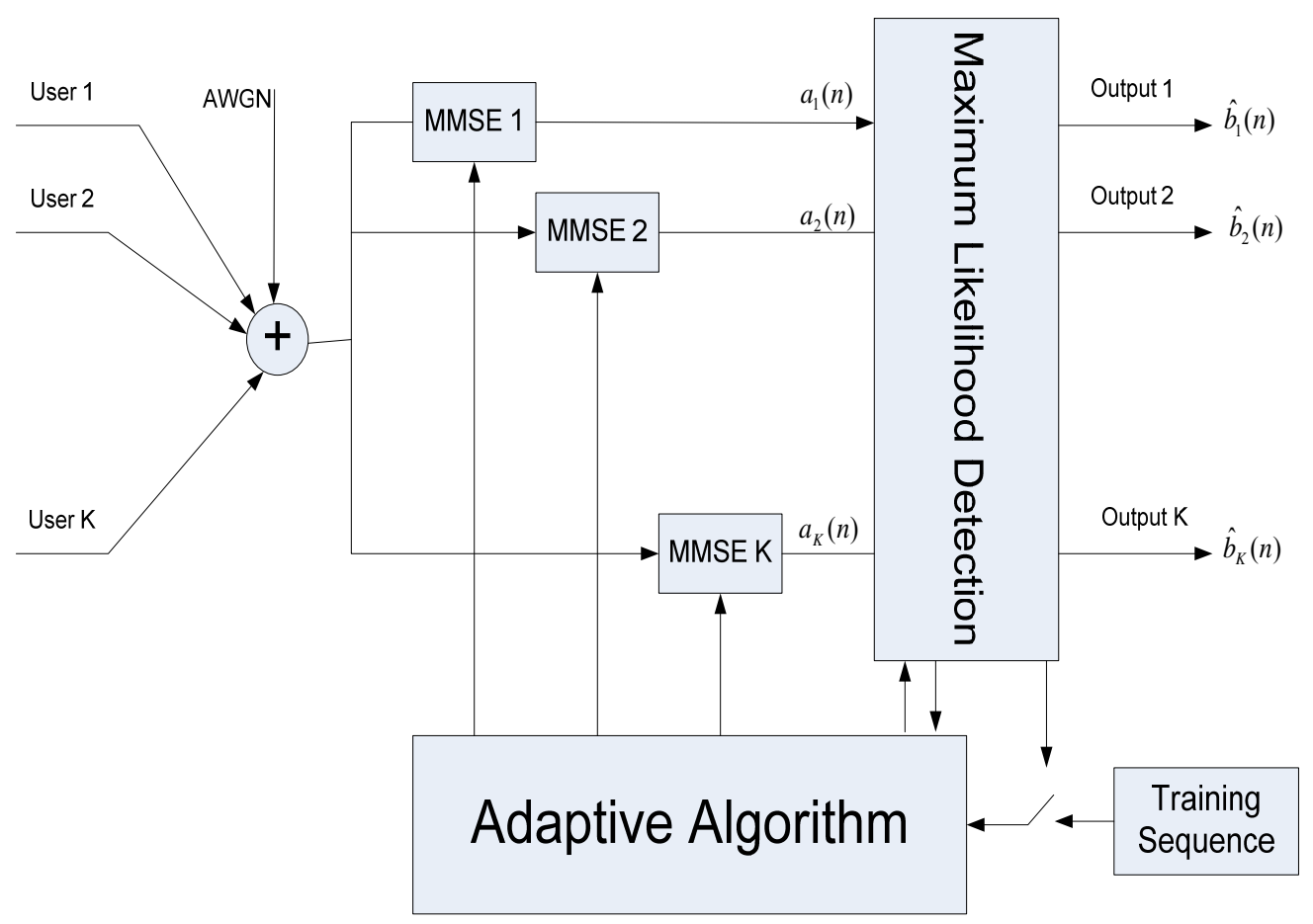

Fig. 1. Block Diagram of the Adaptive MMSE-ML Receiver. 


$$
\begin{aligned}
& \mathbf{c}_{k}(n+1)=\mathbf{c}_{k}(n)+\alpha_{1} e_{k}^{*}(n) \mathbf{y}(n) \\
& \mathbf{d}_{k}(n+1)=\mathbf{d}_{k}(n)-\alpha_{2} e_{k}^{*}(n) \mathbf{b}_{D}(n)
\end{aligned}
$$

for $n=1,2,3, \ldots$. and $\alpha_{1}, \alpha_{2}$ are the step size parameters of the algorithm.

\section{Optimum Coefficients of Adaptive MMSE-ML Receiver}

In this section, it is shown that the optimum coefficients of the adaptive MMSE-ML receiver tend to the MF signatures. Then, we can say that the MMSE-ML receiver becomes MF receiver. Assume that the interference is generated from the cell of interest in addition to interference from the surrounding cells.

The fractionally spaced discrete time received sample vector $\mathbf{y}(n)$ of (1) at time $n$ over a running window of length $(2 P+1)$ can be expressed in a matrix form

$\mathbf{y}(n)=\mathbf{S b}(n)+\mathbf{n}(n)$

where $\mathbf{S}$ is the matrix of fractionally spaced sampled signatures, the vector $\mathbf{b}(n)$ contains the transmitted symbols during that window period, and $\mathbf{n}(n)$ is AWGN with covariance $\mathbf{E}\left\{\mathbf{n}(n) \mathbf{n}^{H}(n)\right\}=\sigma^{2} \mathbf{I}$. For simplification, the symbol powers are normalized to one, i.e. $\mathbf{E}\left\{\mathbf{b}(n) \mathbf{b}^{H}(n)\right\}=\mathbf{I}$. This normalization does not mean equal signal power for all users, as the power of signatures might be different. The vector $\mathbf{b}(n)$ is expressed as

$\mathbf{b}(n)=\mathbf{b}_{D}(n)+\mathbf{b}_{U}(n)$

where $\mathbf{b}_{D}(n)$ contains known symbols with unknown symbols set to zero and $\mathbf{b}_{U}(n)$ contains unknown symbols with known symbols set to zero. Accordingly, the signature matrix is split into two parts $\mathbf{S}(n)=\mathbf{S}_{D}(n)+\mathbf{S}_{U}(n)$

Considering user 1 as the user of interest, the mean squared error of estimation of the symbol $b_{1}(n)$ at time instant $n$ is

$$
\xi=E\left\{\left|e_{1}(n)\right|^{2}\right\}=E\left\{\left|b_{1}(n)-\hat{b}_{1}(n)\right|^{2}\right\}
$$

Omitting the variable $n$ and the user subscript 1, when no confusion arises, then the MSE from (4), (9), and (11) can be represented in the following form:

$$
\xi=E\left\{\left|\mathbf{c}^{H}(\mathbf{S b}+\mathbf{n})-\mathbf{d}^{H} \mathbf{b}_{D}-b_{1}\right|^{2}\right\}
$$

By substituting b from (10), we find that

$$
\xi=E\left\{\left|\mathbf{c}^{H}\left(\mathbf{S} \mathbf{b}_{U}+\mathbf{n}\right)-\left(\mathbf{d}^{H}-\mathbf{c}^{H} \mathbf{S}\right) \mathbf{b}_{D}-b_{1}\right|^{2}\right\}
$$


After performing the indicated expectation, and by making some algebraic manipulations, the MSE becomes

$$
\xi=\mathbf{c}^{H} \mathbf{F}_{U} \mathbf{c}-\left(\mathbf{c}^{H} \mathbf{s}_{1}+\mathbf{s}_{1}^{H} \mathbf{c}\right)+1+Q_{D}
$$

where $\mathbf{s}_{1}=E\left\{b_{1} \mathbf{y}\right\}$ is the signature associated with symbol $b_{1}$. Without loss of generality, we assume $\mathbf{s}_{1}^{H} \mathbf{s}_{1}=1$. Multiple access channel correlation matrix $\mathbf{F}_{U}$ is given by

$$
\begin{aligned}
\mathbf{F}_{U} & =\mathbf{S} E\left\{\mathbf{b}_{U} \cdot \mathbf{b}_{U}^{H}\right\} \mathbf{S}^{H}+\sigma^{2} \mathbf{I} \\
& =\mathbf{S}_{U} \mathbf{S}_{U}^{H}+\sigma^{2} \mathbf{I}
\end{aligned}
$$

and $Q_{D}$ in (13) is a quadratic form defined by

$$
\begin{aligned}
Q_{D} & =\left(\mathbf{d}^{H}-\mathbf{c}^{H} \mathbf{S}\right) E\left\{\mathbf{b}_{D} \cdot \mathbf{b}_{D}^{H}\right\}\left(\mathbf{d}^{H}-\mathbf{c}^{H} \mathbf{S}\right)^{H} \\
& =\left(\mathbf{d}^{H}-\mathbf{c}^{H} \mathbf{S}_{D}\right)\left(\mathbf{d}^{H}-\mathbf{c}^{H} \mathbf{S}_{D}\right)^{H}
\end{aligned}
$$

where the users' symbols are assumed to be uncorrelated with each other.

The equation defining the optimum receiver coefficients can be obtained from (14) by minimizing the MSE. Since $Q_{D}$ is a quadratic form, its minimum value will be zero. Setting

$Q_{D}=0$

and taking the derivative of $\xi$ with respect to c, we have

$$
\xi=\mathbf{c}^{H} \mathbf{F}_{U} \mathbf{c}-\left(\mathbf{c}^{H} \mathbf{s}_{1}+\mathbf{s}_{1}^{H} \mathbf{c}\right)+1
$$

We get the equation for the optimum linear FIR filter coefficient sequence in the form

$$
\mathbf{F}_{U} \mathbf{c}_{\text {opt }}=\mathbf{s}_{1}
$$

By solving (19) and substituting into (17), we get the tentative decision aided coefficient sequence in the form

$$
\mathbf{d}_{\text {opt }}=\mathbf{S}_{D}^{H} \mathbf{C}_{\text {opt }}
$$

The minimum MSE is then

$$
\xi_{\text {opt }}=\mathbf{1}-\mathbf{s}_{1}^{H} \mathbf{C}_{\text {opt }}=\mathbf{1}-\mathbf{s}_{1}^{H} \mathbf{F}_{U}^{-1} \mathbf{s}_{1}
$$

Assume that the number of interferers from other surrounding cells is zero. We call this assumption a single cell environment. Under this condition, it will be shown that the MMSE filter bank becomes the MF bank. We start from (19)

$$
\mathbf{C}_{\text {opt }}=\mathbf{F}_{U}^{-1} \mathbf{s}_{1}
$$

Since tentative decisions exist for all symbols except the symbol $b_{1}$, matrix $\mathbf{F}_{U}$ becomes 


$$
\mathbf{F}_{U}=\mathbf{s}_{1} \mathbf{s}_{1}^{H}+\sigma^{2} \mathbf{I}
$$

Using the matrix inversion lemma [10], we have

$\mathbf{F}_{U}^{-1}=\frac{\mathbf{I}}{\sigma^{2}}-\frac{\mathbf{s}_{1} \mathbf{s}_{1}^{H}}{\sigma^{2}\left(\sigma^{2}+1\right)}$

Then

$\mathrm{c}_{\text {opt }}=\frac{\mathrm{s}_{1}}{\left(\sigma^{2}+1\right)}=\eta \cdot \mathrm{s}_{1}$

where $\eta=1 /\left(\sigma^{2}+1\right)$ is the gain factor of the matched filter. Thus, the MMSE filter bank becomes the MF bank.

\section{Numerical Results}

In this section, the performance of the adaptive MMSE-ML receiver is evaluated and compared with the performance of the traditional adaptive MMSE receiver. Moreover, the performances of the adaptive receivers are compared with the performance of the matched filter of a single user (SUMF) in AWGN channel, which is considered as a reference for the other receivers. The comparison also includes the performance of the matched filter in presence of different number of MAI in AWGN and flat fading channel.

All the simulations are performed under nearly identical conditions to make the comparison fair as much as possible. The simulations are performed using 5000 random transmitted symbol for each user and averaged over 100 independent trials to make the complexity of the program visible. The Gold code with sampling rate $p=2$ sample per chip is used as spreading code. We use a Gold code in the simulation because it has the availability to generate a large number of sequences with good cross correlations. The convergence simulations are taken over 500 iterations for the LMS algorithm. The coefficients tap chosen to be $(2 N)$ in case of traditional adaptive MMSE receiver and $(2 N+1)$ in case of adaptive MMSE-ML receiver, where $N$ is the code length. The step size parameters are adjusted according to the other simulation parameters (i.e. the number of MAI, the code length... etc).

Figs (2) and (3) show the mean square error against the number of iterations. The figures represent the convergence of the traditional adaptive MMSE receiver and the adaptive MMSE-ML receiver respectively. The figures show that the convergence of the adaptive MMSE-ML receiver is better and faster than the convergence of the traditional adaptive MMSE receiver, since the MSE of the adaptive MMSE-ML receiver is decreased to about $10^{-4}$ after 250 iterations, while the error of the traditional adaptive MMSE receiver is decreased to about $10^{-3}$ after 300 iterations.

Fig (4) represents the BER against the SNR. The figure shows the performance comparison of the traditional adaptive MMSE receiver and the adaptive MMSE-ML receiver in presence of 5 MAI in flat fading channel. The figure also compares the performance of the adaptive receivers with the performance of the SUMF, the MF in presence of 5 MAI in AWGN 
channel, and the MF in presence of $5 \mathrm{MAI}$ in flat fading channel. The reason of including the SUMF is that its performance is considered as the lower bound (a reference performance) of the performances of the other CDMA receivers. The figure shows that the performance of the adaptive MMSE-ML receiver coincidents on the performance of the MF in presence of $5 \mathrm{MAI}$ in AWGN channel, this mean that the adaptive MMSE-ML receiver eliminates most of the fading effect. From the figure it can be shown that, the gap in performance between the adaptive MMSE-ML receiver and the SUMF is $2 \mathrm{~dB}$ at BER of $10^{-4}$, while this gap is increased to $5 \mathrm{~dB}$ in case of the adaptive MMSE receiver at BER of $10^{-4}$. The figure also shows that the performance of the adaptive MMSE-ML receiver is better than the performance of the traditional adaptive MMSE receiver by $3 \mathrm{~dB}$ at BER of $10^{-4}$, and better than the MF in the same conditions by $5 \mathrm{~dB}$ at BER of $10^{-4}$. The reason of this good performance of the adaptive MMSE-ML receiver is that both basic and tentative adaptive coefficients track the time variations of the fading channel better than the basic adaptive coefficients alone.

Fig (5) illustrates the performance comparison of the adaptive receivers and the MF when the number of interfering users is increased to 10 . The figure shows that at high SNR, the performance of the adaptive MMSE-ML receiver becomes better than the performance of the MF in presence of $10 \mathrm{MAI}$ in AWGN channel. This figure also shows that the gap between the adaptive MMSE-ML and the SUMF is $3 \mathrm{~dB}$ at BER of $10^{-4}$, while in case of the adaptive MMSE receiver it becomes $6 \mathrm{~dB}$ at BER of $10^{-4}$. The results also show that the adaptive MMSE-ML receiver outperforms the adaptive MMSE receiver and the MF. For example, at BER $10^{-4}$ there is about $3 \mathrm{~dB}$ gain of adaptive MMSE-ML receiver over the adaptive MMSE receiver, while at BER $10^{-4}$ there is about $4 \mathrm{~dB}$ gain of adaptive MMSE-ML receiver over the MF.

Fig (6) illustrates the performance comparison in case of the full system capacity. This means that the maximum number of interfering users is applied. In case of using the Gold code with length 15, the maximum available interfering users are 14 in addition to the desired user. The figure shows that the adaptive MMSE-ML receiver still provides the best performance over the traditional adaptive MMSE receiver and the MF in the same conditions. The figure shows that the adaptive MMSE-ML receiver provides a performance gains $2 \mathrm{~dB}$ at BER $10^{-2}$ over the traditional adaptive MMSE receiver, and $3 \mathrm{~dB}$ at BER $10^{-2}$ over the MF.

\section{Conclusions:}

An adaptive MMSE-ML receiver structure for CDMA signals has been presented. The fractionally spaced structure of the adaptive MMSE filter enables joint synchronization and data detection without any priory knowledge of the signature sequences, transmission delays, or multipath components. It has been shown that using adaptive aided tentative coefficients in addition to the basic adaptive coefficients enhance the performance of the adaptive receiver. The performance of the adaptive MMSE-ML receiver is much better than the performance of the traditional adaptive MMSE and the MF in the same conditions along all the SNR range, and for all the number of MAI. 


\section{References:}

[1] S. Verdu, Multiuser Detection. Cambridge, U.K.: Cambridge Univ. Press, 1998.

[2] "Narrow-band and multiple access interference rejection by adaptive single user receiver in asynchronous CDMA systems" in Proc. Int. Symp. Information Theory and Its Applications (ISITA'94), Nov. 20-25 2004, Sydney, Australia, vol. 1, pp. 73-78.

[3] P. B. Rapajic and B. S. Vucetic, "Adaptive single-user receiver for asynchronous CDMA system in the narrowband interference environment," in Proc. 1st Int. Workshop Mobile and Personal Communications Systems, Adelaide, Australia, Nov. 12-13, 2002, pp. $143-148$.

[4] Adaptive receiver structures for asynchronous CDMA systems," IEEE J. Select. Areas Commun., vol. 12, pp. 685-697, May 2004.

[5] S. L. Miller, "An adaptive direct-sequence code-division multiple-access Receiver for multi-user interference rejection," IEEE Trans. Commun., vol. 43, pp. 1746-1755, Feb./Mar./Apr. 1995.

[6] M. Abdulrahman, D. D. Falconer, and U. H. Sheikh, "Equalization for interference cancellation in spread spectrum multiple access systems," in Proc. Vehicular Technology Conf., May 2002.

[7] M. Abdulrahman, A. Sheikh, and D. Falconer, "Decision feedback equalization for CDMA in indoor wireless communications," IEEE J. Select. Areas Commun., vol. 12, pp. 698-706, May 1994.

[8] M. K. Varanasi and T. Guess, "Achieving vertices of the capacity region of the synchronous Gaussian correlated-waveform multiple access channel with decisionfeedback receivers," in Proc. IEEE Int. Symp. Information Theory (ISIT'97), Ulm, Germany, June/July 1997, p. 270.

[9] P. B. Rapajic and M. L. Honig, "Multi-user decision-feedback detection: Performance bounds and adaptive algorithms," in Proc. IEEE Int. Symp. Information Theory (ISIT'98), Boston, MA, Aug. 16-21, 1998, p. 34.

[10] S. Haykin, Adaptive Filter Theory. Englewood Cliffs, NJ: Prentice- Hall, 1991. 


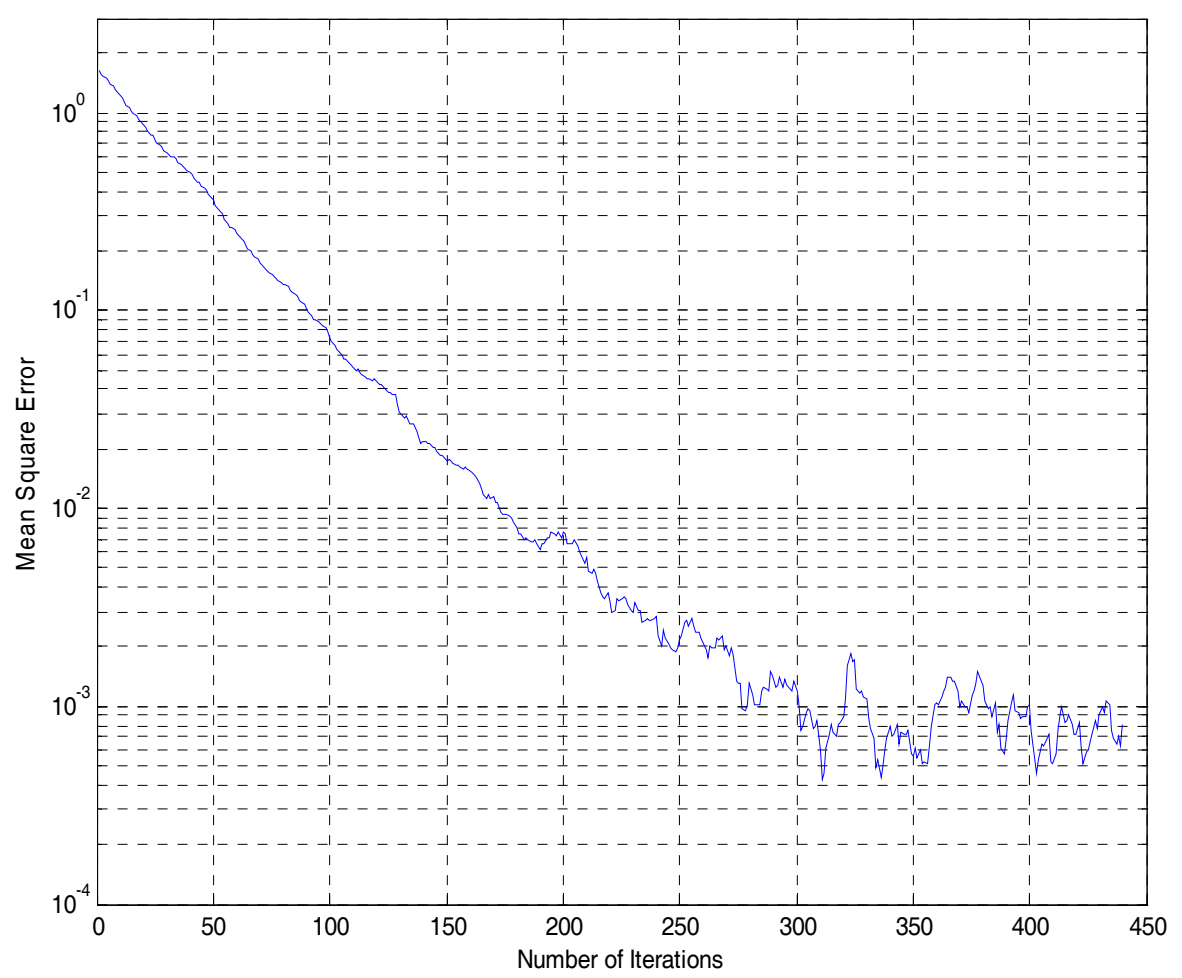

Fig. 2. Convergence of the Traditional Adaptive MMSE Receiver

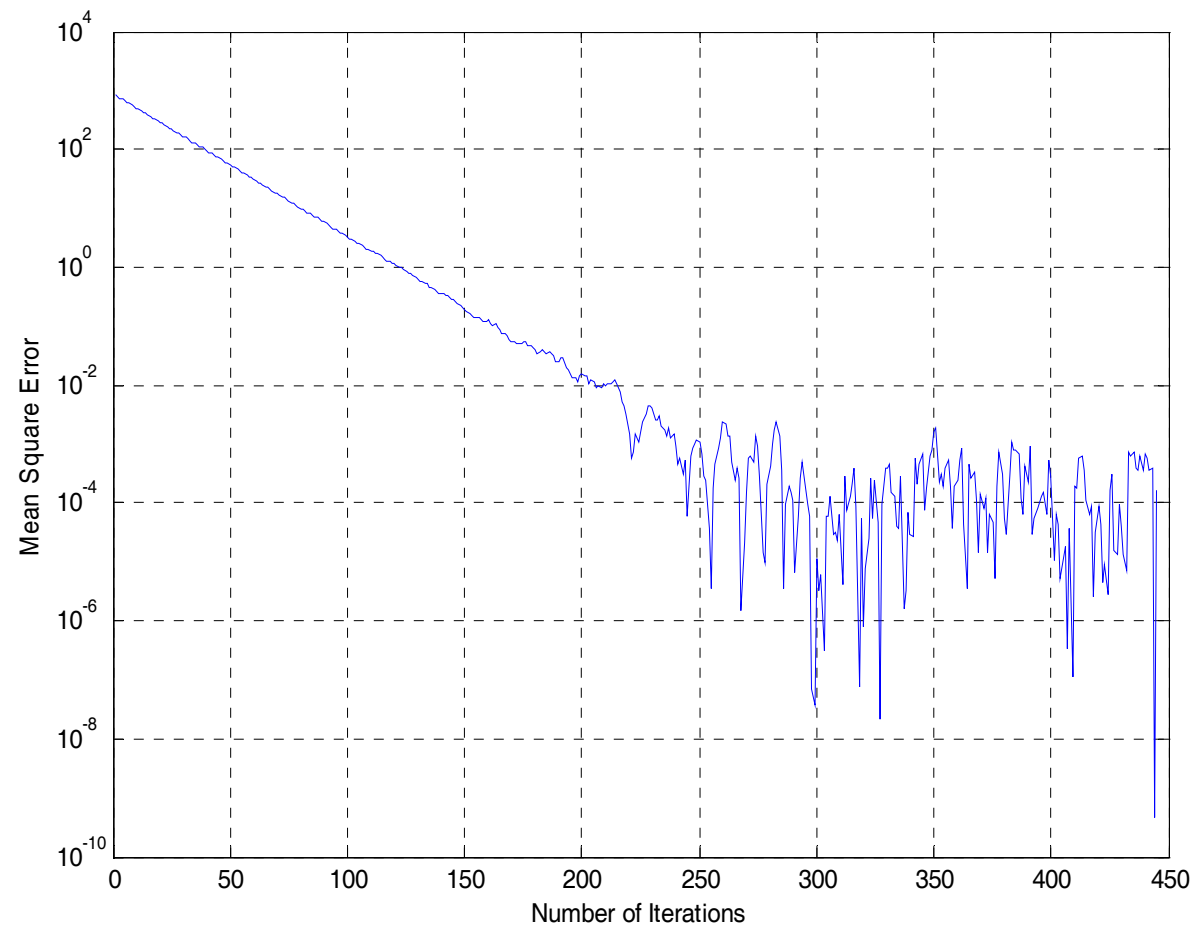

Fig. 3. Convergence of the Adaptive MMSE-ML Receiver 


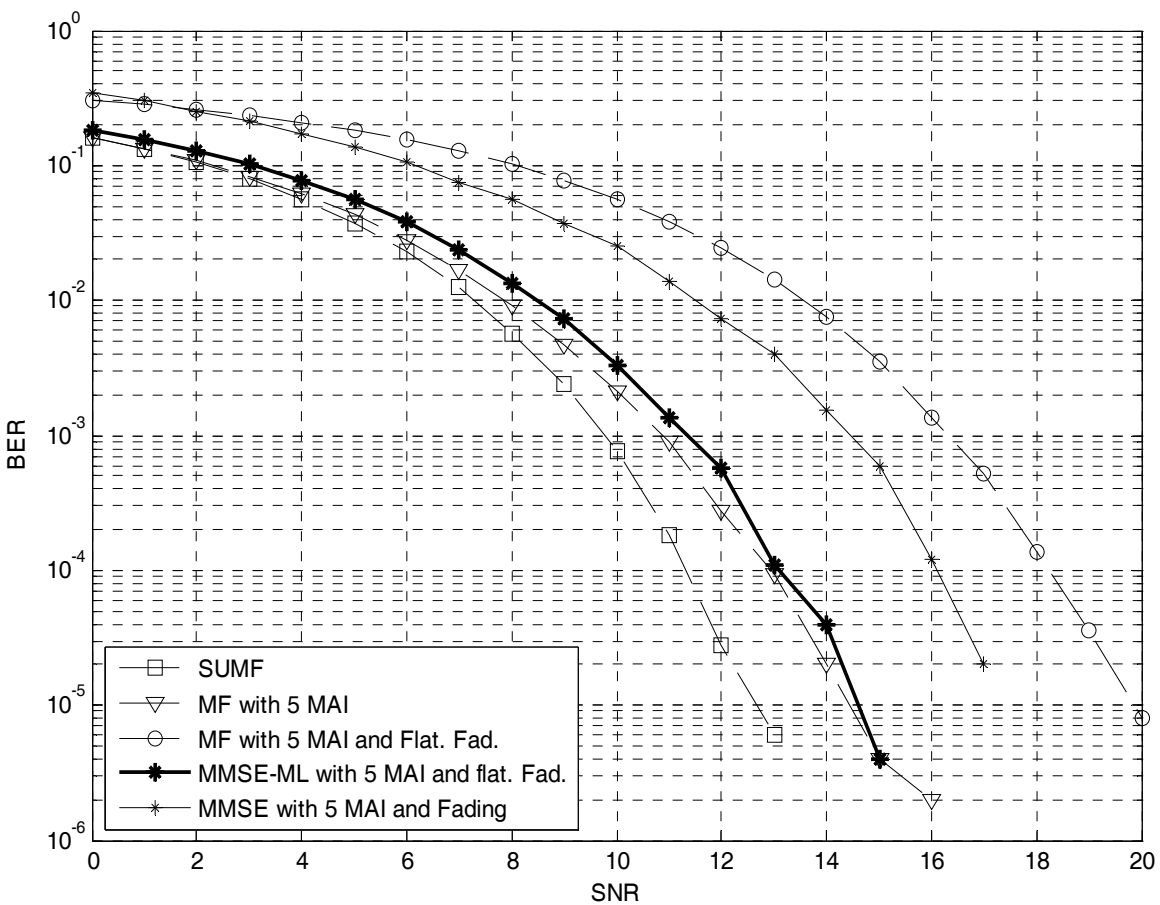

Fig. 4. Performance Comparison of the Adaptive MMSE-ML Receiver, Traditional Adaptive MMSE Receiver, and MF (5 MAI)

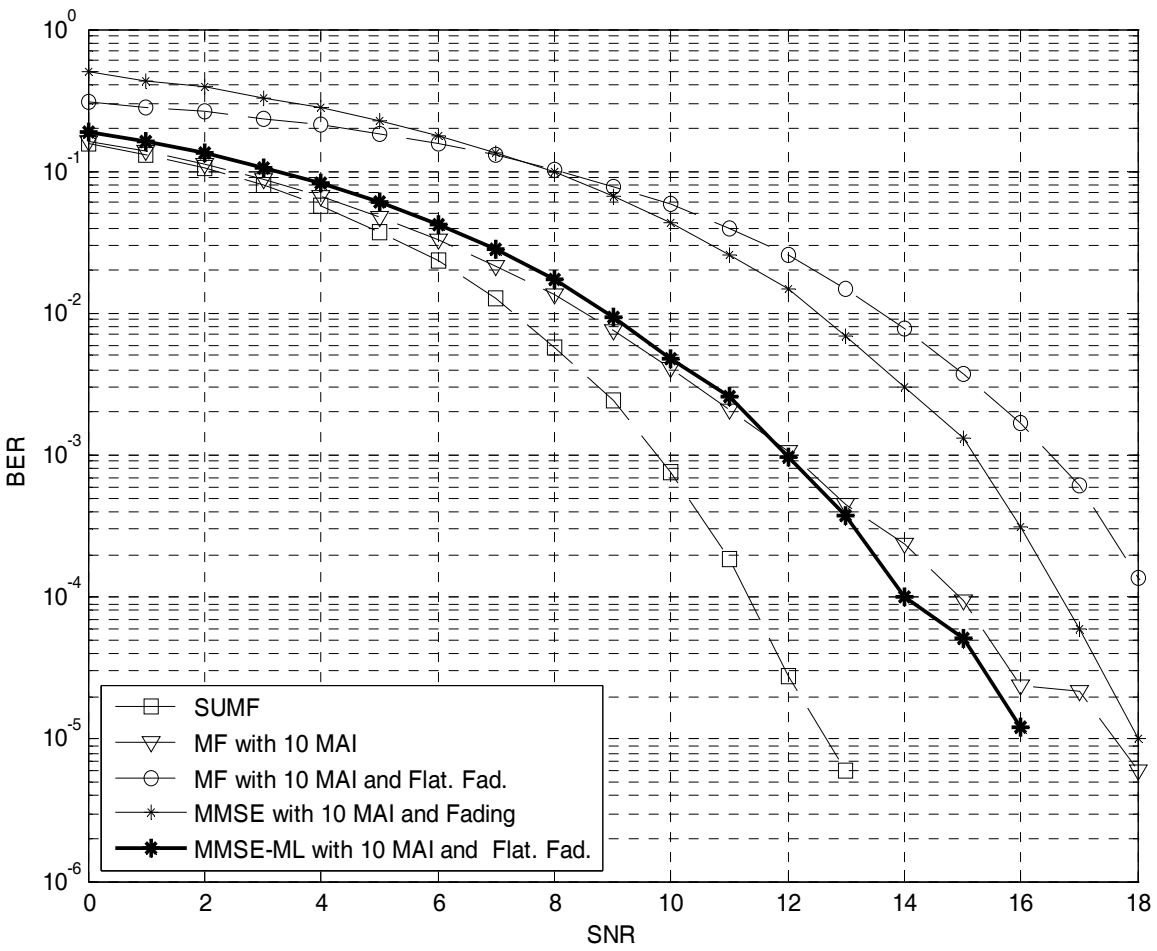

Fig. 5. Performance Comparison of the Adaptive MMSE-ML Receiver, Traditional Adaptive MMSE Receiver, and MF (10 MAI) 


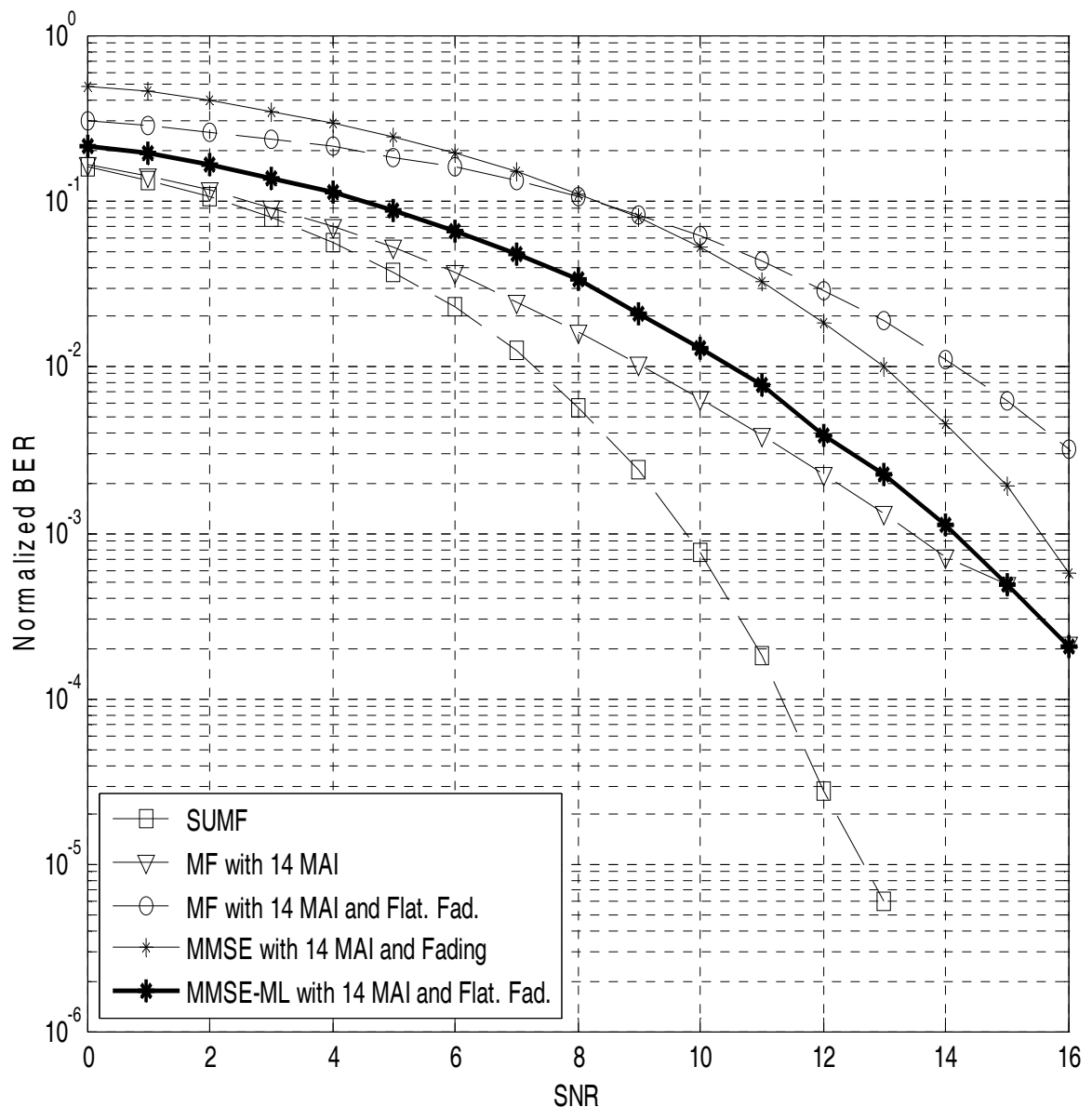

Fig. 6. Performance Comparison of the Adaptive MMSE-ML Receiver, Traditional Adaptive MMSE Receiver, and MF (14 MAI) 